\title{
Interfering Channel Estimation for Radar and Communication Coexistence
}

\author{
Fan Liu*, Adrian Garcia-Rodriguez ${ }^{\dagger}$, Christos Masouros* and Giovanni Geraci ${ }^{\ddagger}$ \\ * Department of Electronic and Electrical Engineering, University College London, London, UK \\ ${ }^{\dagger}$ Nokia Bell Labs, Dublin 15, Ireland \\ $\ddagger$ Universitat Pompeu Fabra, Barcelona, Spain \\ Email: \{fan.liu, c.masouros\}@ucl.ac.uk, a.garciarodriguez.2013@ieee.org, dr.giovanni.geraci@gmail.com
}

\begin{abstract}
We investigate the interfering channel estimation in radar and communication coexistence, where a multi-input-multioutput (MIMO) radar is operated in a "search and track" mode, and a MIMO base station (BS) is attempting to acquire the interfering channel state information (ICSI) between them, which is required for the precoding designs. In contrast to conventional training based techniques, we exploit radar probing waveforms as pilot signals, which requires no coordination between the systems. As the radar randomly transmits searching and tracking waveforms, it is challenging for the BS to directly obtain the ICSI. We therefore propose a Rao test approach to firstly identify the working mode of the radar, and then estimate the channel. We further provide theoretical performance analysis for the Rao detector. Finally, we assess the effectiveness of the proposed approach by numerical simulations, which show that the BS is able to estimate the ICSI with limited information from the radar.
\end{abstract}

\section{INTRODUCTION}

Radar systems are nowadays deployed in various frequency bands worldwide, with extensive usage on environment sensing, navigation, surveillance and localization, etc. Below $6 \mathrm{GHz}$, radar applications are allocated primary use of a significant portion of the spectrum, e.g., airborne navigation radars close to the $3.4 \mathrm{GHz}$ band, shipborne and Vessel Traffic Service (VTS) radar at $5.6 \mathrm{GHz}$. It is worth noting that these bands have seen increasing cohabitation with commercial wireless systems such as LTE and Wi-Fi. With the allocation of available spectrum to newer communication technologies, the interference in radar bands is on the rise, and has raised concerns from governmental and military organizations on the safeguarding of critical radar operations [1]. Accordingly, there is a growing interest into reliable solutions to enable the spectral coexistence of communication and radar transmission.

By exploiting the multi-antenna nature of modern communication and radar systems, existing approaches mainly focus on designing precoders to eliminate the mutual interference between the two systems. In [2], the radar signals are precoded by a so-called null-space precoder, which is formulated via singular value decomposition (SVD), thus the interference generated to the BS can be zero-forced. To further improve the system performance, the works of [3], [4] have employed optimization based techniques to design the precoders and waveforms at either radar or communication's side, such that certain performance metrics can be optimized.

While the aforementioned methods are well-designed, they require the knowledge of the interfering channel between the radar and the BS. Such information would have to be acquired by sending known training symbols from the TX to the RX [2], or via the coordination of a control center connected to radar and communication systems [3]. It is worth noting that these methods require the full cooperation between both systems, which inevitably occupy extra computational and signaling resources of the radar. Moreover, since it is the cellular operator who exploits the spectrum of the radar, it is the performance of the latter that should be primarily guaranteed, i.e., the radar resources should be allocated to target detection rather than obtaining the ICSI.

In this paper, we consider a more practical line of work that involves the interfering channel estimation in the coexistence of a MIMO BS and a MIMO radar performing "search and track", where the BS estimates the ICSI by exploiting the radar probing waveforms as the pilot signals. The radar is assumed to be agnostic to the interference or even the operation of the BS. Due to the stochastic motion of the target, the radar randomly transmits searching and tracking waveforms during each pulse repetition interval (PRI), which makes it difficult for the BS to acquire the ICSI. To resolve this issue, a Rao test based framework is proposed to identify the working mode of the radar before estimating the channel, followed by a comprehensive performance analysis. Finally, simulation results are provided to validate our theoretical analysis, showing that the proposed approach is effective in obtaining the ICSI between the two systems.

\section{SYSTEM MODEL}

As shown in Fig. 1, we consider a MIMO radar that is detecting targets located in the far field. For simplicity, we assume that the MIMO radar employs an $M$-antenna array for both transmission and reception. Meanwhile, an $N$-antenna BS operating in the same frequency band is receiving interference from the radar and trying to acquire the ICSI between them. Below we provide the system models for both the radar and the BS.

\section{A. Radar Signal Transmission - Search and Track}

It is widely known that by employing incoherent waveforms, the MIMO radar achieves higher Degrees of Freedom (DoFs) and better performance than the conventional phased-array 


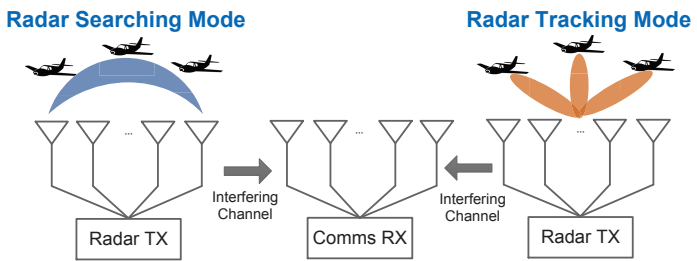

Fig. 1. MIMO radar and BS coexistence.

radar that transmits correlated waveforms [5]. By denoting the MIMO radar probing waveform as $\mathbf{X} \in \mathbb{C}^{M \times L}$, its spatial covariance matrix can be given as [5]

$$
\mathbf{R}_{X}=\frac{1}{L} \mathbf{X X}^{H}
$$

where $L$ is the length of the radar pulse. Throughout the paper we consider $L \geq N \geq M$, and assume uniform linear arrays (ULA) at both the radar and the BS. The corresponding beampattern can be thus given in the form [5]

$$
P_{d}(\theta)=\mathbf{a}^{H}(\theta) \mathbf{R}_{X} \mathbf{a}(\theta),
$$

where $\theta$ denotes the azimuth angle, and $\mathbf{a}(\theta)=$ $\left[1, e^{j 2 \pi \Delta \sin (\theta)}, \ldots, e^{j 2 \pi(M-1) \Delta \sin (\theta)}\right]^{T} \in \mathbb{C}^{M \times 1}$ is the steering vector of the transmit antenna array with $\Delta$ being the antenna spacing normalized by the wavelength.

When the orthogonal waveform is transmitted by the MIMO radar, it follows that [6], [7]

$$
\mathbf{R}_{X}=\frac{P_{R}}{M} \mathbf{I}_{M}
$$

where $P_{R}$ is the total transmit power of the radar, and $\mathbf{I}_{M}$ is the $M$-dimensional identity matrix. It is easy to see from (2) that the covariance matrix (3) generates an omni-directional beampattern, which is typically used for searching when there is limited information about the target locations [5]. Once a target is detected, the radar switches to the tracking mode, where it will no longer transmit orthogonal waveforms and will generate a directional beampattern that points to the specific location, thus obtaining a more accurate observation. This, however, results in a non-orthogonal transmission, i.e., $\mathbf{R}_{X} \neq \frac{P_{R}}{M} \mathbf{I}_{M}$. Given the random motion of the target, we assume in this paper that the MIMO radar changes its probing waveform $\mathbf{X}$ randomly within each PRI, which makes it challenging for the BS to estimate the interfering channel between them.

\section{B. BS Signal Reception Model}

Denoting the interfering channel as $\mathbf{G} \in \mathbb{C}^{N \times M}$, the received signal matrix at the $\mathrm{BS}$ can be given as

$$
\mathbf{Y}=\mathbf{G X}+\mathbf{Z}
$$

where $\mathbf{Z}=\left[\mathbf{z}_{1}, \mathbf{z}_{2}, \ldots, \mathbf{z}_{L}\right] \in \mathbb{C}^{N \times L}$ is the noise matrix, with $\mathbf{z}_{l} \sim \mathcal{C N}\left(\mathbf{0}, N_{0} \mathbf{I}_{N}\right), \forall l$. In the proposed hypothesis testing framework, the noise power $N_{0}$ plays an important role for normalizing the test statistic. Note that when radar keeps silent, the BS will receive nothing but the noise, and $N_{0}$ can be measured at this stage. Since the radar antenna number and its transmit power are fixed parameters, they can also be easily known to the BS operators. Therefore, it is reasonable to assume that the BS knows the value of $N_{0}, M$ and $P_{R}$.

In order to estimate the channel and the noise power $N_{0}$, the BS needs to know when the radar is transmitting, i.e., it must synchronize its clock with the radar pulses. During one PRI, the radar only transmits for a portion of the time, typically below $10 \%$, and employs the remaining $90 \%$ for receiving, during which the radar remains silent. Such a ratio is called duty cycle. By exploiting this property, the BS is able to blindly estimate the beginning and the end of a radar pulse by some simple methods, such as energy detection. Hence, we adopt the assumption that the BS can perfectly synchronize its clock with the radar pulses, i.e., it is able to know the beginning and the end of each radar pulse.

\section{Proposed Approach Based on RaO Test}

In light of the above discussion, the channel estimation procedure at the BS is to firstly identify the working mode of the radar based on the received radar interference, i.e., whether the radar is searching or tracking, and then estimate the interfering channel by exploiting the limited knowledge about the radar waveforms. In the following, we will develop the approach for the BS to acquire the ICSI when radar is randomly changing its probing waveform.

\section{A. Preliminaries}

Consider the ideal case where the BS knows exactly the waveform sent by the radar in each PRI. Recalling (4), the well-known maximum likelihood estimation (MLE) of the channel $\mathbf{G}$ is given as [8]

$$
\hat{\mathbf{G}}=\mathbf{Y} \mathbf{X}^{H}\left(\mathbf{X X}^{H}\right)^{-1}
$$

which is also known as the Least-Squares estimation (LSE) for $\mathbf{G}$. Since the radar changes its waveform randomly at each PRI, (5) can not be directly applied and it is difficult to estimate the channel directly.

Let us denote the searching and tracking waveforms as $\mathbf{X}_{0}$ and $\mathbf{X}_{1}$, respectively, where $\mathbf{X}_{0}$ is spatially orthogonal. In a realistic scenario, the tracking waveform $\mathbf{X}_{1}$ may vary from pulse to pulse. This is because the target to be detected may move very fast, which results in rapid changes in its parameters such as the distance, velocity and the azimuth angle. Hence, it is far from realistic to assume the BS knows $\mathbf{X}_{1}$. Nevertheless, as an omni-directional searching waveform, there is no reason for $\mathbf{X}_{0}$ to be changed rapidly. Indeed, in some cases, the radar may only use one waveform for omni-searching. Based on the above, to assume that the BS only knows $\mathbf{X}_{0}$ seems to be a more practical choice ${ }^{1}$. This leads to the following hypothesis

\footnotetext{
${ }^{1}$ Note the fact that such information exchange can be easily performed once prior to transmission, since the searching waveform of the radar remains unchanged. In contrast, conventional training based techniques require a much tighter cooperation between both systems.
} 
testing (HT) problem [8]

$$
\begin{aligned}
& \mathcal{H}_{0}: \mathbf{X}=\mathbf{X}_{0}, \mathbf{G}, \\
& \mathcal{H}_{1}: \mathbf{X} \neq \mathbf{X}_{0}, \mathbf{G} .
\end{aligned}
$$

In (6), the channel $\mathbf{G}$ to be estimated is called the nuisance parameter [8].

At first glance, the conventional generalized likelihood ratio test (GLRT) seems to be applicable to (6), which requires the MLE of G under both hypotheses. It can be trivially seen that the MLE of $\mathbf{G}$ under $\mathcal{H}_{0}$ can be obtained by substituting $\mathbf{X}_{0}$ into (5). However, note that to obtain the MLE of $\mathbf{G}$ under $\mathcal{H}_{1}$ is equivalent to solving the following optimization problem

$$
\min _{\mathbf{G}, \mathbf{X}}\|\mathbf{Y}-\mathbf{G X}\|_{F}^{2} \quad \text { s.t. }\|\mathbf{X}\|_{F}^{2}=L P_{R},
$$

where the constraint is to ensure the power budget of the radar-transmitted waveform. While the above problem is nonconvex, it yields trivial zero solutions with a high probability. This is because the problem (7) is likely to have more than enough degrees of freedom to ensure a zero objective function, since the channel matrix is unconstrained, and the norm constraint can be always satisfied by scaling $\mathbf{X}$, where the scaling factor can be incorporated in G. Therefore, the likelihood function under $\mathcal{H}_{1}$ will always be greater than that of $\mathcal{H}_{0}$, which makes the HT design meaningless.

\section{B. Proposed Rao Test}

Realizing the fact above, we propose to use the Rao test (RT) to solve the HT problem (15), which does not need the MLE under $\mathcal{H}_{1}$. Based on [9]-[11], let us define

$$
\boldsymbol{\Theta}=\left[\operatorname{vec}^{T}(\mathbf{X}), \operatorname{vec}^{T}(\mathbf{G})\right]^{T} \triangleq\left[\boldsymbol{\theta}_{r}^{T}, \boldsymbol{\theta}_{s}^{T}\right]^{T} .
$$

Then, the RT statistic for the complex-valued parameters can be given in the form

$T_{R}(\mathbf{Y})$

$=\left.\left.2 \frac{\partial \ln p(\mathbf{Y} ; \boldsymbol{\Theta})}{\partial \operatorname{vec}(\mathbf{X})}\right|_{\boldsymbol{\Theta}=\tilde{\Theta}} ^{T}\left[\mathbf{J}^{-1}(\tilde{\mathbf{\Theta}})\right]_{\boldsymbol{\theta}_{r} \boldsymbol{\theta}_{r}} \frac{\partial \ln p(\mathbf{Y} ; \boldsymbol{\Theta})}{\partial \operatorname{vec}^{*}(\mathbf{X})}\right|_{\boldsymbol{\Theta}=\tilde{\Theta}} \underset{H_{0}}{\stackrel{H_{1}}{\gtrless}} \gamma$,

where $\tilde{\boldsymbol{\Theta}}=\left[\boldsymbol{\theta}_{r}^{T}, \hat{\boldsymbol{\theta}}_{s}^{T}\right]^{T}=\left[\operatorname{vec}^{T}\left(\mathbf{X}_{0}\right), \operatorname{vec}^{T}\left(\hat{\mathbf{G}}_{0}\right)\right]^{T}$ is the MLE under $\mathcal{H}_{0}$, and $\left[\mathbf{J}^{-1}(\tilde{\boldsymbol{\Theta}})\right]_{\boldsymbol{\theta}_{r} \boldsymbol{\theta}_{r}}$ is the upper-left partition of $\mathbf{J}^{-1}(\tilde{\boldsymbol{\Theta}})$, with $\mathbf{J}(\boldsymbol{\Theta})$ being the Fisher Information Matrix (FIM). Substituting $\mathbf{X}_{0}$ into (5), it follows that

$$
\hat{\mathbf{G}}_{0}=\mathbf{Y} \mathbf{X}_{0}^{H}\left(\mathbf{X}_{0} \mathbf{X}_{0}^{H}\right)^{-1}=\frac{M}{L P_{R}} \mathbf{Y} \mathbf{X}_{0}^{H} .
$$

It should be highlighted that the Rao test only lets the BS determine if the radar is using the searching mode, i.e., whether the searching waveform $\mathbf{X}_{0}$ is transmitted in the current radar PRI. In that case, the BS could obtain the MLE of the channel by use of (10). Otherwise, the BS is required to wait until an orthogonal waveform is transmitted by the radar.

\section{Closed-form Expression of the Rao Detector}

To analyze the performance of the proposed approach, it is necessary to derive the closed-form expression of the Rao detector. First of all, note that the probability density function (PDF) of the received signal matrix can be given as

$$
\begin{aligned}
& p(\mathbf{Y}) \\
& =\left(\pi N_{0}\right)^{-N L} \exp \left(-\frac{1}{N_{0}} \operatorname{tr}\left((\mathbf{Y}-\mathbf{G X})^{H}(\mathbf{Y}-\mathbf{G X})\right)\right)
\end{aligned}
$$

The logarithm PDF (log-PDF) can be accordingly obtained as

$$
\ln p=-N L \ln \pi N_{0}-\frac{1}{N_{0}} \operatorname{tr}\left((\mathbf{Y}-\mathbf{G X})^{H}(\mathbf{Y}-\mathbf{G X})\right) \text {. }
$$

According to [8], the FIM can be partitioned as

$$
\mathbf{J}(\boldsymbol{\Theta})=\left[\begin{array}{ll}
\mathbf{J}_{r r} & \mathbf{J}_{r s} \\
\mathbf{J}_{s r} & \mathbf{J}_{s s}
\end{array}\right] .
$$

where we have

$$
\begin{gathered}
\mathbf{J}_{r r}=\mathbb{E}\left(\frac{\partial \ln p}{\partial \operatorname{vec}^{*}(\mathbf{X})} \frac{\partial \ln p}{\partial \operatorname{vec}^{T}(\mathbf{X})}\right), \\
\mathbf{J}_{s s}=\mathbb{E}\left(\frac{\partial \ln p}{\partial \operatorname{vec}^{*}(\mathbf{G})} \frac{\partial \ln p}{\partial \operatorname{vec}^{T}(\mathbf{G})}\right), \\
\mathbf{J}_{r s}=\mathbb{E}\left(\frac{\partial \ln p}{\partial \operatorname{vec}^{*}(\mathbf{X})} \frac{\partial \ln p}{\partial \operatorname{vec}^{T}(\mathbf{G})}\right)=\mathbf{J}_{s r}^{H},
\end{gathered}
$$

By some algebraic computations, the FIM can be expressed as

$$
\mathbf{J}(\boldsymbol{\Theta})=\frac{4}{N_{0}}\left[\begin{array}{cc}
\mathbf{I}_{L} \otimes \mathbf{G}^{T} \mathbf{G}^{*} & \mathbf{X}^{H} \otimes \mathbf{G}^{T} \\
\mathbf{X} \otimes \mathbf{G}^{*} & \mathbf{X X}^{H} \otimes \mathbf{I}_{N}
\end{array}\right] .
$$

By recalling the definition of $\tilde{\boldsymbol{\Theta}}$, and noting that $\mathbf{X}_{0} \mathbf{X}_{0}^{H}=$ $\frac{L P_{R}}{M} \mathbf{I}_{M} \triangleq \rho \mathbf{I}_{M}$, we have

$$
\begin{aligned}
& {\left[\mathbf{J}^{-1}(\tilde{\boldsymbol{\Theta}})\right]_{\theta_{r} \theta_{r}}=\left(\mathbf{J}_{r r}(\tilde{\boldsymbol{\Theta}})-\mathbf{J}_{r s}(\tilde{\mathbf{\Theta}}) \mathbf{J}_{s s}^{-1}(\tilde{\mathbf{\Theta}}) \mathbf{J}_{s r}(\tilde{\mathbf{\Theta}})\right)^{-1}} \\
& =\frac{N_{0}}{4}\left(\left(\mathbf{I}_{L}-\frac{1}{\rho} \mathbf{X}_{0}^{H} \mathbf{X}_{0}\right) \otimes\left(\hat{\mathbf{G}}_{0}^{T} \hat{\mathbf{G}}_{0}^{*}\right)\right)^{-1}
\end{aligned}
$$

where $\rho=\frac{L P_{R}}{M}$, and $\hat{\mathbf{G}}_{0}$ is given by (10). By using (9), (10) and (18), the Rao test statistic can be expressed as

$$
T_{R}(\mathbf{Y})=\frac{2}{N_{0}} \operatorname{tr}\left(\begin{array}{c}
\left(\mathbf{I}_{L}-\frac{M}{L P_{R}} \mathbf{X}_{0}^{H} \mathbf{X}_{0}\right) \mathbf{Y}^{H} \mathbf{Y} \mathbf{X}_{0}^{H} \\
\times\left(\mathbf{X}_{0} \mathbf{Y}^{H} \mathbf{Y} \mathbf{X}_{0}^{H}\right)^{-1} \mathbf{X}_{0} \mathbf{Y}^{H} \mathbf{Y}
\end{array}\right) \stackrel{\underset{\mathcal{H}_{0}}{\gtrless}}{\stackrel{\mathcal{H}_{1}}{\gtrless}}
$$

\section{A Special Case}

It is clear from (19) that we do not need any information about $\mathbf{X}_{1}$ for solving the HT problem (6), which makes it a suitable detector for the practical scenario where the BS only knows $\mathbf{X}_{0}$. Nevertheless, it is very difficult to analytically derive the CDF of (19) due to the highly non-linear operations involved. Here we only focus on a special case where the distribution becomes tractable. Note that if $L \geq M=N$ holds true, $\mathbf{Y X}_{0}^{H} \in \mathbb{C}^{N \times N}$ and $\mathbf{X}_{0} \mathbf{Y}^{H} \in \mathbb{C}^{N \times N}$ become the 
invertible square matrices with a high probability, in which case we have

$$
\begin{aligned}
& \mathbf{Y} \mathbf{X}_{0}^{H}\left(\mathbf{X}_{0} \mathbf{Y}^{H} \mathbf{Y} \mathbf{X}_{0}^{H}\right)^{-1} \mathbf{X}_{0} \mathbf{Y}^{H} \\
& =\left(\left(\mathbf{X}_{0} \mathbf{Y}^{H}\right)^{-1} \mathbf{X}_{0} \mathbf{Y}^{H} \mathbf{Y} \mathbf{X}_{0}^{H}\left(\mathbf{Y} \mathbf{X}_{0}^{H}\right)^{-1}\right)^{-1}=\mathbf{I}_{N} .
\end{aligned}
$$

It follows that

$$
\begin{aligned}
& T_{R s}(\mathbf{Y})=\frac{2}{N_{0}} \operatorname{tr}\left(\left(\mathbf{I}_{L}-\frac{M}{L P_{R}} \mathbf{X}_{0}^{H} \mathbf{X}_{0}\right) \mathbf{Y}^{H} \mathbf{Y}\right) \\
& =\frac{2}{N_{0}} \operatorname{tr}\left(\mathbf{Y}\left(\mathbf{I}_{L}-\frac{M}{L P_{R}} \mathbf{X}_{0}^{H} \mathbf{X}_{0}\right) \mathbf{Y}^{H}\right) \underset{\mathcal{H}_{0}}{\stackrel{\mathcal{H}_{1}}{\gtrless}} \gamma
\end{aligned}
$$

is the Rao detector under this special case. It can be seen that (21) is a quadratic form in Gaussian variables. The matrix $\mathbf{P} \triangleq \mathbf{I}_{L}-\frac{M}{L P_{R}} \mathbf{X}_{0}^{H} \mathbf{X}_{0}$ is a projection matrix, which projects any vector to the null-space of $\mathbf{X}_{0}^{H}$. Therefore, we have

$$
\operatorname{tr}\left(\mathbf{G X}_{1} \mathbf{P} \mathbf{X}_{1}^{H} \mathbf{G}^{H}\right) \geq 0=\operatorname{tr}\left(\mathbf{G X}_{0} \mathbf{P} \mathbf{X}_{0}^{H} \mathbf{G}^{H}\right) .
$$

The above equation (22) can be viewed as the hypothesis testing for the noise-free scenario, where we see that the Rao detector (21) is effective in differentiating the two hypotheses. By adding the Gaussian noise to $\mathbf{G X}_{1}$ and $\mathbf{G X}_{0}$, it can be inferred that $T_{R s}\left(\mathbf{Y} ; \mathcal{H}_{1}\right) \geq T_{R s}\left(\mathbf{Y} ; \mathcal{H}_{0}\right)$ with a high probability in the high SNR regime, which makes the detector (21) valid.

According to [8], (21) subjects to non-central chi-squared distribution under both hypotheses. Under $\mathcal{H}_{0}$, the noncentrality parameter is given by

$$
\mu_{0}=\frac{2}{N_{0}} \operatorname{tr}\left(\mathbf{G X}_{0}\left(\mathbf{I}_{L}-\frac{M}{L P_{R}} \mathbf{X}_{0}^{H} \mathbf{X}_{0}\right) \mathbf{X}_{0}^{H} \mathbf{G}^{H}\right)=0
$$

which indicates that $T_{R s}\left(\mathbf{Y} ; \mathcal{H}_{0}\right)$ is in fact central chi-squared distributed. Under $\mathcal{H}_{1}$, the non-centrality parameter is

$$
\mu=\frac{2}{N_{0}} \operatorname{tr}\left(\mathbf{G X}_{1}\left(\mathbf{I}_{L}-\frac{M}{L P_{R}} \mathbf{X}_{0}^{H} \mathbf{X}_{0}\right) \mathbf{X}_{1}^{H} \mathbf{G}^{H}\right) .
$$

The DoFs of the two distributions are given by

$$
\begin{aligned}
& K=2 \operatorname{rank}\left(\mathbf{I}_{N} \otimes \mathbf{P}\right) \\
& =2 N \operatorname{rank}(\mathbf{P})=2 N \operatorname{tr}(\mathbf{P})=2 N(L-M),
\end{aligned}
$$

where we use the property of the idempotent matrix that $\operatorname{rank}(\mathbf{P})=\operatorname{tr}(\mathbf{P})$.

Following the above, $T_{R s}$ satisfies that

$$
T_{R s} \sim\left\{\begin{array}{l}
\mathcal{H}_{0}: \mathcal{X}_{K}^{2}, \\
\mathcal{H}_{1}: \mathcal{X}_{K}^{2}(\mu) .
\end{array}\right.
$$

Hence, the decision error probability for the special Rao detector (21) is given by

$$
P_{R s}=\left(1-\mathcal{F}_{\mathcal{X}_{K}^{2}}(\gamma)\right) P\left(\mathcal{H}_{0}\right)+\mathcal{F}_{\mathcal{X}_{K}^{2}(\mu)}(\gamma) P\left(\mathcal{H}_{1}\right),
$$

where $\mathcal{F}_{\mathcal{X}_{K}^{2}}$ and $\mathcal{F}_{\mathcal{X}_{K}^{2}(\mu)}$ are the CDFs of central and noncentral chi-squared distributions, respectively, and $P\left(\mathcal{H}_{i}\right), i=$ 0,1 denotes the a priori probabilities of the two hypotheses.

\section{Channel Estimation Performance}

By denoting the estimated channel as $\hat{\mathbf{G}}=$ $\mathbf{Y} \mathbf{X}^{H}\left(\mathbf{X X}^{H}\right)^{-1}$, the squared error can be given in the form

$$
\begin{aligned}
& \phi=\|\hat{\mathbf{G}}-\mathbf{G}\|_{F}^{2}=\left\|\mathbf{Y} \mathbf{X}^{H}\left(\mathbf{X} \mathbf{X}^{H}\right)^{-1}-\mathbf{G}\right\|_{F}^{2} \\
& =\left\|\left(\mathbf{X X}^{H}\right)^{-1} \mathbf{X} \mathbf{Y}^{H}-\mathbf{G}^{H}\right\|_{F}^{2} .
\end{aligned}
$$

Let us define

$$
\begin{aligned}
& \overline{\mathbf{y}}=\operatorname{vec}\left(\mathbf{Y}^{H}\right) \sim \mathcal{C N}\left(\operatorname{vec}\left(\mathbf{X}^{H} \mathbf{G}^{H}\right), N_{0} \mathbf{I}_{N L}\right), \\
& \mathbf{T}=\mathbf{I}_{N} \otimes\left(\mathbf{X X}^{H}\right)^{-1} \mathbf{X}, \overline{\mathbf{g}}=\operatorname{vec}\left(\mathbf{G}^{H}\right) .
\end{aligned}
$$

Then, (28) can be simplified as

$$
\phi=\|\mathbf{T} \overline{\mathbf{y}}-\overline{\mathbf{g}}\|^{2} .
$$

Based on basic statistics and linear algebra, we also have

$$
\mathbf{y}_{e q} \triangleq \mathbf{T} \overline{\mathbf{y}}-\overline{\mathbf{g}} \sim \mathcal{C N}\left(\mathbf{0}, N_{0} \mathbf{T} \mathbf{T}^{H}\right),
$$

where

$$
\begin{aligned}
& \mathbf{T} \mathbf{T}^{H}=\mathbf{I}_{N} \otimes\left(\mathbf{X X}^{H}\right)^{-1} \mathbf{X} \cdot \mathbf{I}_{N} \otimes \mathbf{X}^{H}\left(\mathbf{X X}^{H}\right)^{-1} \\
& =\mathbf{I}_{N} \otimes\left(\mathbf{X X}^{H}\right)^{-1} .
\end{aligned}
$$

Based on the above, the MSE of the channel estimation can be obtained as

$$
\begin{aligned}
& \mathbb{E}(\phi)=\mathbb{E}\left(\left\|\mathbf{y}_{e q}\right\|^{2}\right)=\mathbb{E}\left(\operatorname{tr}\left(\mathbf{y}_{e q} \mathbf{y}_{e q}^{H}\right)\right)=\operatorname{tr}\left(\mathbb{E}\left(\mathbf{y}_{e q} \mathbf{y}_{e q}^{H}\right)\right) \\
& =N_{0} \operatorname{tr}\left(\mathbf{I}_{N} \otimes\left(\mathbf{X X}^{H}\right)^{-1}\right)=\frac{N_{0} N}{L} \operatorname{tr}\left(\mathbf{R}_{X}^{-1}\right) .
\end{aligned}
$$

In the case that $\mathbf{X}_{0}$ is employed for channel estimation, the MSE can be given as

$$
\mathbb{E}(\phi)=\frac{N_{0} N}{L} \operatorname{tr}\left(\left(\frac{P_{R}}{M} \mathbf{I}_{M}\right)^{-1}\right)=\frac{N_{0} M^{2} N}{L P_{R}} .
$$

\section{Numerical Results}

In this section, numerical results are provided to verify the effectiveness of the proposed approaches. We use $\mathbf{X}_{0}=\sqrt{\frac{L P_{R}}{M}} \mathbf{U}$ as the radar searching waveform, where $\mathbf{U} \in \mathbb{C}^{M \times L}$ is an arbitrarily given unitary matrix. For the tracking waveform $\mathbf{X}_{1}$, we firstly solve the classic $3 \mathrm{~dB}$ beampattern design problem to obtain the waveform covariance matrix $\mathbf{R} \in \mathbb{C}^{M \times M}$, which is given in [5]. We then obtain the tracking waveform $\mathbf{X}_{1}$ by the Cholesky decomposition of $\mathbf{R}$. The mainlobe of the radar focuses on the angle of $0^{\circ}$, and the desired $3 \mathrm{~dB}$ beamwidth is $10^{\circ}$.

Since the optimal threshold for Rao test is difficult to obtain, we provide the ergodic empirical thresholds, which are computed by Monte Carlo simulations with a large number of channel realizations, and can guarantee that the average error probability is minimized. Meanwhile, we also compute the optimal threshold that corresponds to one single channel realization for $M=N$, where the theoretical error probability is given in (27). Note that such a threshold is not obtainable in practical scenarios, as it requires the BS to know the channel 


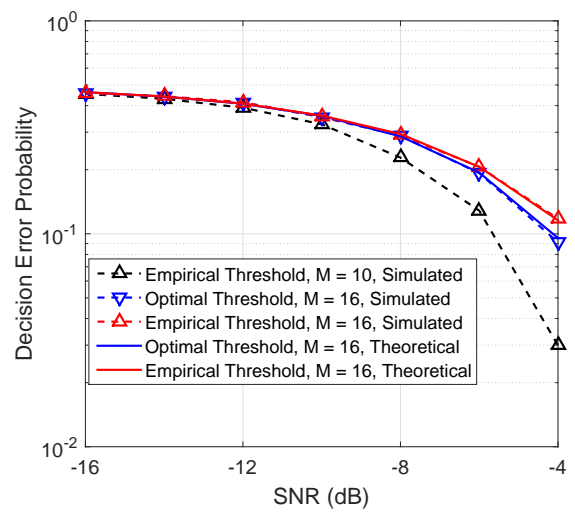

Fig. 2. Error probability vs. SNR. $M=\{10,16\}, N=16, L=20$.

a priori. In our simulations, the optimal threshold serves as the performance benchmark for the Rao test. For simplicity, we assume that the probability that radar is operating in searching mode is $50 \%$, i.e., $P\left(\mathcal{H}_{0}\right)=P\left(\mathcal{H}_{1}\right)=0.5$. Without loss of generality, we set $P_{R}=1$, and define the transmit SNR of radar as $\mathrm{SNR}=P_{R} / N_{0}$. Unless otherwise specified, we fix $L=20$, and assume half-wavelength separation between adjacent antennas. Finally, we adopt a Rayleigh fading channel $\mathbf{G}$, i.e., the entries of $\mathbf{G}$ are independent and identically distributed (i.i.d.) and subject to the standard complex Gaussian distribution.

We firstly show in Fig. 2 the detection performance for the proposed approach, where we fix $N=16$, and set $M=10$ and $M=16$ respectively. Since the analytical performance for the nonequal-antenna case is intractable, we only show the performance with empirical threshold for $M=10$, which is computed by Monte Carlo simulations. It can be observed that the theoretical curves match well with the simulated ones, which proves the correctness of our performance analysis. When $M=10$, the performance of the Rao detector is superior to that of the case of $M=16$, which is sensible given that the BS exploits more DoFs for hypothesis testing in the former case.

We study the channel estimation performance in Fig. 3, where we fix the radar antenna number as $M=5$, and increase the BS antennas from $N=4$ to $N=20$. Note that the hypothesis testing exploits the power of all the entries in the received signal matrix to make the binary decision, which does not require a high SNR per entry to guarantee a successful outcome. This is very similar to the concept of diversity gain. Nevertheless, for estimating the channel, we need to estimate each entry individually, where the diversity gain does not exist. For this reason, we fix the SNR at $15 \mathrm{~dB}$ to achieve the normal estimation performance. It can be seen from Fig. 3 that the theoretical curves match well with the simulated ones, which proves the correctness of (33) and (34). Secondly, the MSE increases with the rise of the BS antenna number. This is because the number of the matrix entries to be estimated is increasing while the power available for estimation is fixed.

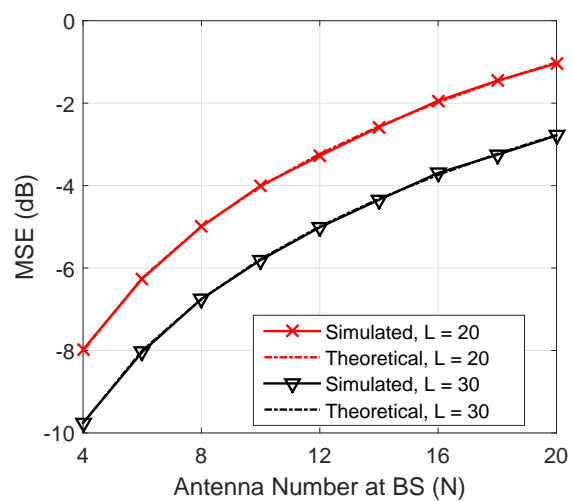

Fig. 3. MSE vs. number of antennas at the BS. $M=5, \mathrm{SNR}=15 \mathrm{~dB}$.

By further dividing (34) by $M N$, we see that the MSE per entry keeps unchanged.

\section{CONCLUSION}

In this paper, we have proposed an interfering channel estimation method for radar and cellular coexistence, where we assume that the radar switches randomly between the searching and tracking modes, and the BS is attempting to estimate the radar-cellular interfering channel by use of the radar probing waveforms. To acquire the channel state information, the BS firstly identifies the working mode of the radar by use of Rao test, and then estimates the channel parameters. Our simulations show that the theoretical curves match well with the numerical results, and that the BS can effectively differentiate the radar operation modes and estimate the channel.

\section{REFERENCES}

[1] DARPA. (2016) Shared spectrum access for radar and communications (SSPARC). [Online]. Available: http://www.darpa.mil/program/sharedspectrum-access-for-radar-and-communications

[2] J. A. Mahal, A. Khawar, A. Abdelhadi, and T. C. Clancy, "Spectral coexistence of MIMO radar and MIMO cellular system," IEEE Trans. Aerosp. Electron. Syst., vol. 53, no. 2, pp. 655-668, Apr 2017.

[3] B. Li and A. P. Petropulu, "Joint transmit designs for coexistence of MIMO wireless communications and sparse sensing radars in clutter," IEEE Trans. Aerosp. Electron. Syst., vol. 53, no. 6, pp. 2846-2864, Dec 2017.

[4] F. Liu, C. Masouros, A. Li, T. Ratnarajah, and J. Zhou, "MIMO radar and cellular coexistence: A power-efficient approach enabled by interference exploitation," IEEE Trans. Signal Process., vol. 66, no. 14, pp. 36813695, Jul 2018.

[5] J. Li and P. Stoica, "MIMO radar with colocated antennas," IEEE Signal Process. Mag., vol. 24, no. 5, pp. 106-114, Sept 2007.

[6] — MIMO Radar Signal Processing. John Wiley \& Sons, 2008.

[7] S. Sun and A. P. Petropulu, "Waveform design for MIMO radars with matrix completion," IEEE J. Sel. Topics Signal Process., vol. 9, no. 8, pp. 1400-1414, Dec 2015.

[8] S. M. Kay, Fundamentals of statistical signal processing. Englewood Cliffs, NJ, USA: Prentice Hall, 1998.

[9] S. M. Kay and Z. Zhu, "The complex parameter Rao test," IEEE Trans. Signal Process., vol. 64, no. 24, pp. 6580-6588, Dec 2016.

[10] W. Liu, J. Liu, L. Huang, D. Zou, and Y. Wang, "Rao tests for distributed target detection in interference and noise," Elsevier Signal Process., vol. 117, pp. 333-342, 2015.

[11] W. Liu, Y. Wang, and W. Xie, "Fisher information matrix, Rao test, and Wald test for complex-valued signals and their applications," Elsevier Signal Process., vol. 94, pp. 1-5, 2014. 